\title{
Experience of Ultrasound-Guided Vacuum-Assisted Excision of a Phyllodes Tumor Arising From Ectopic Axillary Breast Tissue: A Case Report
}

\author{
Go Eun Yang (iD ${ }^{1,}{ }^{*}$ and Soo Jin Kim $^{2}$ \\ ${ }^{1}$ Department of Radiology, Kangwon National University Hospital, 200-702, ChunCheon, South Korea \\ ${ }^{2}$ Department of Radiology, New Korea Hospital, Kyungkido, South Korea \\ Corresponding author: Department of Radiology, Kangwon National University Hospital, 200-702, ChunCheon, South Korea. Email: yangke00@hanmail.net \\ Received 2021 April 04; Revised 2021 September 06; Accepted 2021 September 11.
}

\begin{abstract}
An axillary breast is a common condition, which can cause various diseases arising from the breast tissue. Among these diseases, phyllodes tumors are very rare fibroepithelial tumors, which resemble fibroadenomas. Surgical removal is the treatment of choice for phyllodes tumors to prevent recurrence. Inpatient treatment and general anesthesia are required for surgical removal, and fibrosis, skin scarring, and neurological symptoms may occur after surgery. Ultrasound-guided vacuum-assisted excision, because of its advantages, is an alternative to surgical excision for clinically benign breast masses. Therefore, in cases of clinically benign or concordant benign lesions, following a core needle biopsy, ultrasound-guided vacuum-assisted excision can be considered with sufficient preoperative measures and close follow-up of the ectopic axillary breast.
\end{abstract}

Keywords: Breast, Biopsy, Image-Guided Biopsy, Phyllodes, Axilla

\section{Introduction}

An accessory breast tissue, with a prevalence of 0.6 to $6 \%$, is caused by the failed regression of the primitive mammary tissue. Phyllodes tumors in the ectopic axillary breast tissue are very rare fibroepithelial tumors, and only few cases have been reported in the literature (1-4). Surgical removal is the treatment of choice for phyllodes tumors to prevent recurrence. However, inpatient treatment and general anesthesia are required for the surgical removal of tumors, and fibrosis, skin scarring, and neurological symptoms may occur following surgery. Recently, ultrasound-guided vacuum-assisted excision has been increasingly performed as an alternative to surgical excision for clinically benign breast masses. Therefore, in clinically benign or concordant benign lesions, ultrasound-guided vacuum-assisted excision can be considered after a core needle biopsy with sufficient preoperative measures and close follow-up of the ectopic axillary breast.

\section{Case Presentation}

A 35-year-old woman presented to our clinic with a palpable left axillary mass. She had no history or family history of malignancy. There was a 2.2-cm, ovoid-shaped, lobulating, contoured, hypoechoic mass in the ultrasonographic (US) examination, and the accessory breast tissue was observed around it. Therefore, the tumor was presumed to be a suspicious mass, originating from the accessory breast (Figure 1A). The US-guided 14G core needle biopsy revealed a fibroepithelial tumor with typical multifocal ductal epithelial hyperplasia (Figure 1B). To remove the mass, the patient underwent a US-guided vacuumassisted excision, and the pathological report revealed a benign phyllodes tumor in the accessory breast (Figure 1C).

After one month, there was no definite recurrence or residual mass in the US examination (Figure 1D). However, after one year, a small mass (a suspected recurrent mass) appeared (Figure 1E). Therefore, the patient underwent USguided vacuum-assisted excision for the second time. After three years, there were no abnormalities, such as recurrent masses in the axillary area on mammography (Figures $1 \mathrm{~F}$ ).

\section{Discussion}

Phyllodes tumors are uncommon fibroepithelial breast tumors, accounting for $0.3-1.0 \%$ of all breast neoplasms. The mammographic and sonographic features 

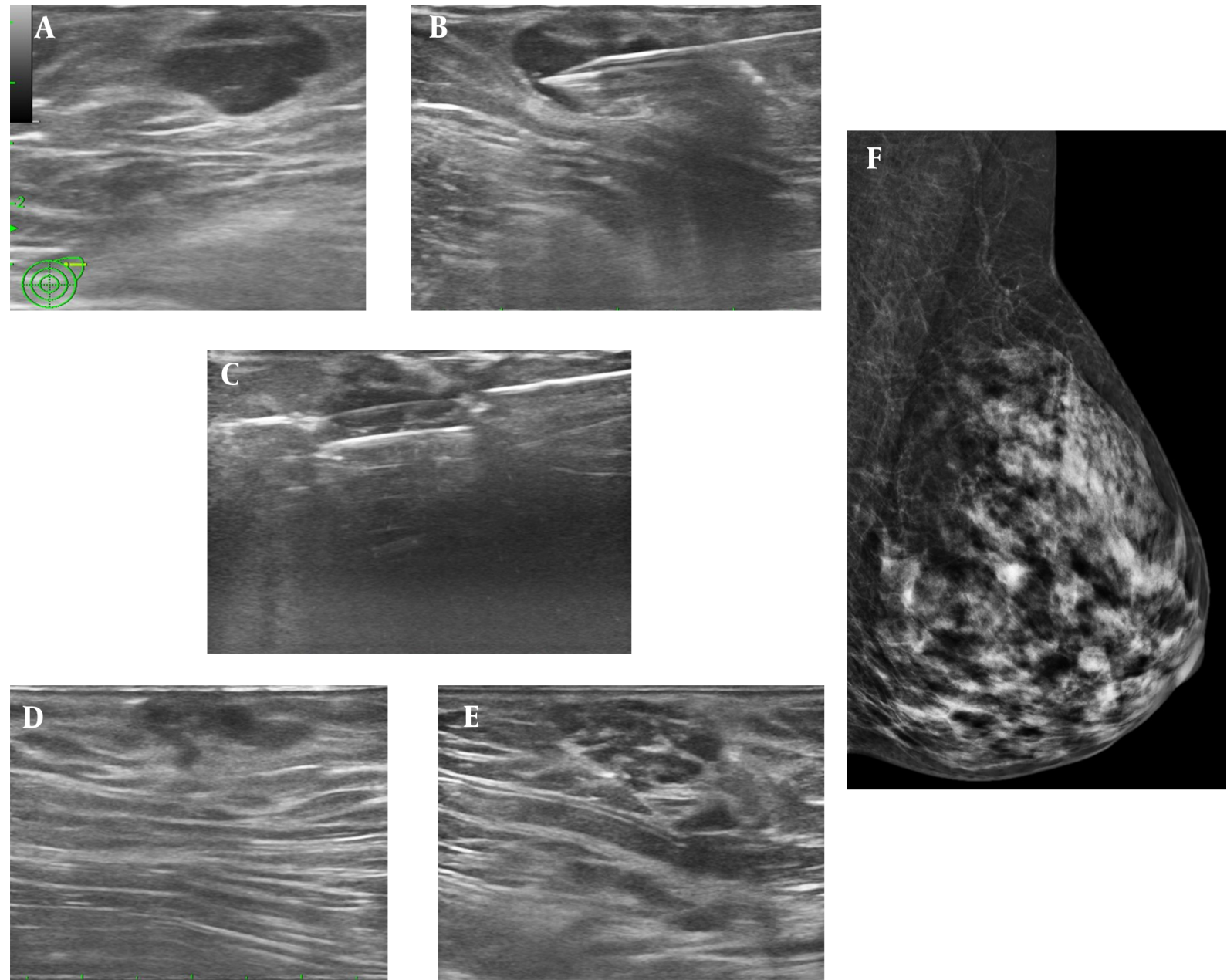

Figure 1. A35-year-old female with a palpable left axillary mass. A, The initial ultrasonography (US) shows an oval, lobulating, hypoechoic mass. B, US-guided core needle biopsy reveals a fibroepithelial tumor with typical multifocal ductal epithelial hyperplasia. C, US-guided vacuum-assisted excision and pathological report of a benign phyllodes tumor in the accessory breast. D, After one month, there was no definite recurrence or residual mass in the US examination. E, After one year, a small mass suspected of recurrence appeared. F, After three years, the mediolateral oblique view of the mammogram showed no axillary mass.

are usually similar to those of pectoral breast lesions $(1-3,5)$. These tumors are uncommon fibroepithelial tumors that resemble fibroadenomas, but differ in terms of local recurrence and metastatic behaviors. Therefore, surgical removal is the treatment of choice for phyllodes tumors to prevent recurrence after an accurate diagnosis (2). Considering the shortcomings of surgery, US-guided vacuum-assisted excision can be an alternative treatment, as in our case, if only local resection is sufficient.

This is the first case report of a phyllodes tumor removal via US-guided vacuum-assisted excision. There was no recurrence or complication during the three-year follow-up after treatment. Accessory breast disease may be asymptomatic or manifest as a palpable mass. It can be removed surgically, although surgery is only necessary if there is a problem. The possibility of disease in the accessory breast tissue should be considered and investigated in the same manner as the pectoral breast tissue (3). For benign and asymptomatic lesions, conservative management is sufficient, although complete excision of symptomatic lesions can be considered $(6,7)$. Overall, US-guided vacuum-assisted excision is performed as an alternative to surgical excision for clinically benign breast masses, such as fibroadenomas, or concordant benign masses after core needle biopsy (8).

There are reports on the treatment of lymph nodes (9), gynecomastia, and fibroadenoma (10) in the axillary area. However, to the best of our knowledge, there is no report on the vacuum-assisted removal of axillary phyllodes tumors. Overall, the complete removal of an axillary mass us- 
ing US-guided vacuum-assisted biopsy may be limited. This is due to the fact that the axillary breast tissue is very close to the skin, and therefore, masses are also very close to the skin; conseuqnetly, a portion of the mass may remain due to skin damage. A safer complete removal is expected if an experienced expert performs the procedure carefully under US guidance.

Regarding the tumor size, more than $96 \%$ of bengin masses $<3 \mathrm{~cm}$ were removed (11). However, among axillary lesions, only smaller masses are prefered for removal. It should be noted that a smaller size is associated with a higher likelihood of complete removal. Also, in general fibroadenomas, even if some of the tissue remains after removal, the probability of recurrence is not high. However, in phyllodes tumors, recurrence is more likely if complete removal is not achieved, and the surgical margin is not secured; these coditions should be well understood, and a sufficient safety margin must be ensured when removing a phyllodes tumor. Besides, the patient should be clearly informed. Nevertheless, the ability to remove masses without scars in a short period for outpatients is an important goal that has not been achieved yet.

In conlusion, after a core needle biopsy for clinically benign or concordant benign lesions, if tumor removal is necessary, US-guided vacuum-assisted excision can be considered with sufficient preoperative measures and close follow-up of the ectopic axillary breast.

\section{Footnotes}

Authors' Contribution: Go Eun Yang majorly contributed to writing this manuscript. Soo Jin Kim substantially contributed to the conception and design of this study. All authors read and approved the final manuscript.

Conflict of Interests: The authors declare no conflicts of interest.

Funding/Support: None declared by authors.
Informed Consent: Written informed consent was obtained from each participant.

\section{References}

1. Saleh HA, Klein LH. Cystosarcoma phyllodes arising synchronously in right breast and bilateral axillary ectopic breast tissue. Arch Pathol Lab Med.1990;114(6):624-6.

2. Oshida K, Miyauchi M, Yamamoto N, Takeuchi T, Suzuki M, Nagashima $\mathrm{T}$, et al. Phyllodes tumor arising in ectopic breast tissue of the axilla. Breast Cancer. 2003;10(1):82-4. doi:10.1007/BF02967630. [PubMed: 12525768].

3. Lim HS, Kim SJ, Baek JM, Kim JW, Shin SS, Seon HJ, et al. Sonographic Findings of Accessory Breast Tissue in Axilla and Related Diseases. J Ultrasound Med. 2017;36(7):1469-78. doi: 10.7863/ultra.16.06056. [PubMed: 28370098].

4. Teke Z, Kabay B, Akbulut M, Erdem E. Primary infiltrating ductal carcinoma arising in aberrant breast tissue of the axilla: a rare entity. Report of a case. Tumori. 2008;94(4):577-83. [PubMed: 18822697].

5. Kim S, Oh HY, Ry Y. Benign Phyllodes Tumor of the Breast Recurring as a Rapidly Growing Recurrent Malignant Phyllodes Tumor: A Case Report. Iran J Radiol. 2019;16(2). doi: 10.5812/iranjradiol.13062.

6. Youk JH, Kim EK, Kim MJ, Lee JY, Oh KK. Missed breast cancers at US-guided core needle biopsy: How to reduce them. Radiographics. 2007;27(1):79-94. doi: 10.1148/rg.271065029. [PubMed:17235000].

7. Jiang $\mathrm{Y}$, Lan $\mathrm{H}$, Ye Q, Jin $\mathrm{K}$, Zhu M, Hu X, et al. Mammotome ((R)) biopsy system for the resection of breast lesions: Clinical experience in two high-volume teaching hospitals. Exp Ther Med. 2013;6(3):75964. doi: 10.3892/etm.2013.1191. [PubMed: 24137261]. [PubMed Central: PMC3786805].

8. Youk JH, Kim H, Kim EK, Son EJ, Kim MJ, Kim JA. Phyllodes tumor diagnosed after ultrasound-guided vacuum-assisted excision: Should it be followed by surgical excision? Ultrasound Med Biol. 2015;41(3):741-7. doi: 10.1016/j.ultrasmedbio.2014.11.004. [PubMed: 25619780].

9. Britton P, Willsher P, Taylor K, Kilburn-Toppin F, Provenzano E, Forouhi P, et al. Microbubble detection and ultrasoundguided vacuum-assisted biopsy of axillary lymph nodes in patients with breast cancer. Clin Radiol. 2017;72(9):772-9. doi: 10.1016/j.crad.2017.03.011. [PubMed: 28427743].

10. Dialani V, James DF, Slanetz PJ. A practical approach to imaging the axilla. Insights Imaging. 2015;6(2):217-29. doi: 10.1007/s13244-014-03678. [PubMed: 25534139]. [PubMed Central: PMC4376818].

11. Fine RE, Boyd BA, Whitworth PW, Kim JA, Harness JK, Burak WE. Percutaneous removal of benign breast masses using a vacuumassisted hand-held device with ultrasound guidance. Am J Surg. 2002;184(4):332-6. doi: 10.1016/s0002-9610(02)00951-0. 